ARTí́CULO

\title{
Análisis de la pesquería de jaiba en la región sureste del golfo de California, México
}

\author{
Analysis of the crab fishery in the southern Gulf of California, Mexico
}

\section{Gilberto Genaro Ortega-Lizárraga ${ }^{1^{*}}$, Guillermo Rodríguez-Domínguez ${ }^{2}$, Raúl Pérez-González ${ }^{2}$, E. Alberto Aragón-Noriega ${ }^{3}$ y Jaime Edzael Mendivil-Mendoza ${ }^{3}$}

\author{
${ }^{1}$ Centro Regional de Investigación Acuícola y Pesquera, INAPESCA, Calzada Sábalo-Cerritos s/n, Contiguo Estero del Yugo, C.P. \\ 82112 Mazatlán, Sinaloa, México \\ ${ }^{2}$ Facultad de Ciencias del Mar, Universidad Autónoma de Sinaloa, Paseo Claussen S/N, Centro, 82000 Mazatlán, Sinaloa, México \\ ${ }^{3}$ Centro de Investigaciones Biológicas del Noroeste, Unidad Sonora, km 2.35, Camino al Tular, Estero de Bacochibampo, C.P. 85454 \\ Guaymas, Sonora, México \\ *Autor corresponsal: gengil@hotmail.com
}

\begin{abstract}
Economically, the fishery crabs Callinectes arcuatus and C. bellicosus in the state of Sinaloa (southern Gulf of California) are the second most important industry for coastal fishermen after the shrimp fishery. Catching crabs is pursued for most of the year and is suspended only at the beginning of the shrimping season and during the months of closure. This fishery is carried out in different zones of the state of Sinaloa, Mexico, and represents a major income source in the coastal fisheries sector; thus, estimating the parameters $B_{\text {MYS }}$ and MSY are fundamental to the sustainable management of the fishery. A 1993-2012 official catch series for 7 zones of the state of Sinaloa was used to detect possible problems of overexploitation of the resource at different study zones as well as globally. The r-values calculated by the catch-MSY method varied from 0.9 to 1.4 , with k-values between 390 and $14,814 \mathrm{t}$ in MSY 131 and 3,302 $t$ for the analyzed zones, while the global estimation was an $r$ of 0.97 , a k of 32,550 and 7,895 t of MSY. For two of the seven zones and the overall evaluation, the estimated biomass was calculated under the $\mathrm{B}_{\mathrm{MRS}}$, so reducing the catch below the lower limit calculated at $95 \%$ is recommended to permit the population stocks to recover.
\end{abstract}

Key words: Callinectes, fishery, stock, Gulf of California

Resumen.- En el sureste del golfo de California, en el estado de Sinaloa, la pesquería de las jaibas Callinectes arcuatus y C. bellicosus es la segunda más importante para los pescadores ribereños, después de la pesquería del camarón. Su captura se realiza durante casi todo el año, solo se suspende en los meses de su veda y al inicio de la temporada de pesca del camarón. La pesquería se realiza en diferentes zonas del estado y representa una gran fuente de ingresos en el sector de pesca ribereña, por ello la estimación de parámetros como la biomasa en el máximo rendimiento sostenible $\left(\mathrm{B}_{\mathrm{MRS}}\right.$ ) y el máximo rendimiento sostenible (MRS) son fundamentales para su manejo sustentable. En la presente investigación,se utilizó la serie de captura oficial de 1993-2012 para 7 zonas de Sinaloa, México, con la finalidad de detectar posibles problemas de sobre-explotación del recurso. Los valores de tasa de incremento poblacional ( $r$ ) calculados por el método de captura-MRS variaron entre 0,89 y 1,4, con una capacidad de carga ( $k$ ) entre 390 y 14.814 t y un MRS de 131 a 3.302 t para las diversas zonas analizadas, mientras que en el análisis para todo el estado se estimó una $r$ de 0,97, k de 32.550 t y un MRS de 7.895 t. En dos de las siete zonas y en la evaluación global, la biomasa estimada se calculó por debajo de la $\mathrm{B}_{\text {MRS }}$, por lo que se recomienda la reducción de la captura por debajo del límite inferior calculado al $95 \%$, con el objetivo de que el stock pueda regenerarse.

Palabras clave: Callinectes, pesquería, stock, golfo de California

\section{INTRODUCCIÓN}

En el golfo de California se desarrolla una importante pesquería de jaibas del género Callinectes, donde destacan Sinaloa por su producción anual de 9.000 t y Sonora con 4.600 t. Las especies del conjunto conocido como jaibas incluyen a C. toxotes (Ordway, 1863), C. arcuatus (Ordway, 1863) y C. bellicosus (Stimpson, 1859). Las dos últimas son las más importantes en las capturas, con una mayor proporción de C. bellicosus en Sonora. En Sinaloa, la principal zona de captura se registra en la zona centronorte de Sinaloa, en las lagunas costeras denominadas localmente como bahías de Santa María La Reforma, Ohuira-Topolobampo y Agiabampo (Ortega-Lizárraga 2012). En Sinaloa, México, las capturas de jaiba variaron entre 2.000 y $5.000 \mathrm{t}$ entre los años 2000 y 2005 , para el 2007 se incrementaron alrededor de $10.000 \mathrm{t}$, en 2008 alcanzó las 13.071 t. En el 2010, la captura disminuyó a $6.226 \mathrm{t}$ y para el 2011 fue de $6.107 \mathrm{t}$, alcanzando una cifra 
histórica en 2017 con 13.627 t (SAGARPA 2011, 2017). Para los pescadores de ribera, la producción de jaiba ha llegado a superar el volumen de captura de camarón, la especie más importante económicamente, pero la jaiba está disponible todo el año, mientras que las capturas de camarón se reducen a unos cuantos meses. La disminución de las capturas, después del máximo histórico de 2008, motivó a diseñar nuevos esquemas de manejo de la pesquería y se estableció una veda de 3 meses, con el fin de proteger el periodo reproductivo de las especies.

El adecuado manejo de las pesquerías requiere de conocimiento de la dinámica poblacional, para mantener las poblaciones explotadas en niveles cercanos al máximo rendimiento sostenible (MRS) (Martell \& Froese 2012). Sin embargo, ante la escasez de información biológica y pesquera para muchas poblaciones, es necesario considerar el uso de métodos menos demandante de información (Rodríguez-Domínguez et al. 2012, 2014). La captura es relativamente fácil de obtener, pero el esfuerzo pesquero es más difícil de registrar eficientemente, por tal razón, en algunos casos las evaluaciones de poblaciones o las estimaciones de abundancia se basan en los datos de captura por unidad de esfuerzo proporcionados por los pescadores comerciales. Sin embargo, Kennelly \& Scandol (2002) encontraron que, a pesar de contar con extensas series de este tipo de datos, sus sesgos e imprecisiones hacen que los muestreos independientes de la pesca se requieran a menudo para corroborar la evaluación de la población. Con base en estos elementos, Martell \& Froese (2012) presentaron un método simple que utiliza los datos de captura en una serie de tiempo, así como información adicional disponible, con la finalidad de aproximarse al MRS. Este método utiliza un conjunto de combinaciones de $\mathrm{r}$ (tasa de incremento poblacional) y $\mathrm{k}$ (capacidad de carga) factibles para la estimación del MRS. Estos autores realizaron estimaciones de r-k y MRS para 146 stocks y los compararon con valores independientes de MRS para 48 stocks del noreste del Atlántico. Aunque el método fue utilizado principalmente en peces, puede ser aplicado a la pesquería de jaiba en las costas de Sinaloa, tal y como fue utilizado por Rodríguez-Domínguez et al. (2014) para las costas de Sonora y Sinaloa, con series de captura hasta el 2011. Con la finalidad de dar seguimiento y generar mejores herramientas para el manejo adecuado de la pesquería de jaiba (C. arcuatus, $C$. bellicosus y C. toxotes) en Sinaloa, en este estudio se estimó el MRS para 7 zonas del estado de Sinaloa de manera independiente y para todo el estado en general, para detectar posibles efectos espaciales en el stock provocados por las capturas.

\section{MATERIALES Y MÉTODOS}

Los datos utilizados fueron series de tiempo de registros oficiales de captura de jaiba en peso vivo en el estado de Sinaloa, México, desde 1993 a 2012, y clasificados por zona de captura (ordenadas de norte a sur): 1) bahías Jitzamuri, El Colorado, Caracol, Lechuguilla, Santa María y Bacorehuis, 2) bahía Ohuira-Topolobampo, 3) bahía Navachiste, 4) bahía de Santa María La Reforma, 5) bahía Altata y ensenada Pabellones, 6) Conchal y bahía Tempehuaya y 7) Mazatlán (esteros El Delfín y El Yugo), El Rosario, laguna de El Caimanero-Escuinapa y Teacapán (Fig. 1). Para el análisis se utilizó el método de Captura-MRS propuesto por Martell $\&$ Froese (2012), en el cual hay que definir la biomasa del stock al inicio y al final de la serie de tiempo de captura analizada y se expresan como intervalos de proporción de la biomasa virgen $(\mathrm{K})$ (también conocida como capacidad de carga). Para la definición de estos intervalos de biomasa inicial $\left(\lambda_{01}, \lambda_{02}\right)$ y final $\left(\lambda_{1}, \lambda_{2}\right)$, Martell \& Froese (2012) proponen valores con base a la proporción de las capturas al inicio y final de la serie, en relación con la captura máxima registrada. Este método también requiere de la definición de intervalos de valores de la tasa intrínseca de crecimiento poblacional (r) y Martell \& Froese (2012) proponen intervalos de $r$ con base al nivel de resiliencia de la especie, en caso de que no se tenga antecedentes sobre estos valores. Los intervalos de $\mathrm{K}$ se proponen como la captura máxima y 100 veces esta captura máxima. Después se generan pares de datos aleatorios de $\mathrm{r}$ y k considerando una distribución uniforme en el intervalo logarítmico de $\mathrm{r}$ y k:

$$
P(r k) \sim \exp [\operatorname{uniform}(\operatorname{Ln}(l i k), \operatorname{Ln}(l s k))]
$$

Se generaron 100.000 pares de $\mathrm{r}$ y k de manera independiente y aleatoria por cada paso de 0,5 entre el límite inferior y superior de la proporción de biomasa definida al inicio de la serie. Posteriormente, con los valores de r y k y la serie de captura se procedió a estimar la biomasa inicial:

$$
B_{0}=\lambda_{0} k * \exp (v t)
$$

y la siguiente biomasa:

$$
B_{t+1}=\left[B_{t}+r B_{t} *\left(1-\frac{B_{t}}{k}\right)-C_{t}\right] * \exp (v t)
$$

dónde $\mathrm{B}_{\mathrm{t}}$ es la biomasa al tiempo $\mathrm{t}, \mathrm{B}_{\mathrm{t}+1}$ es la biomasa un año después de $t, C_{t}$ es la captura anual en el tiempo $t$ y $\exp (v t)$ es el error de proceso y cuando el valor es 1 se considera error de observación. 


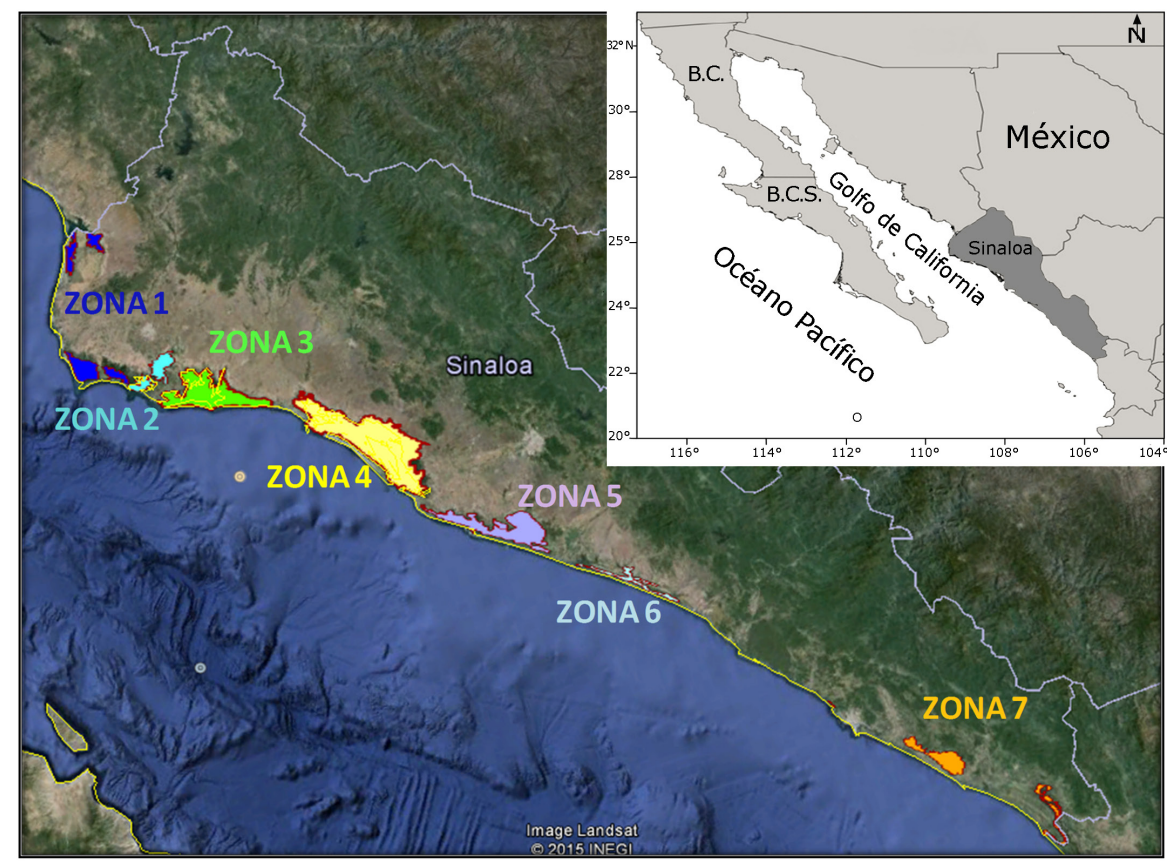

Figura 1. Zonas de captura de jaiba (Callinectes spp.) en el estado de Sinaloa, México / Crab catch areas (Callinectes spp.) in the state of Sinaloa, Mexico

Para el análisis de los datos se consideraron intervalos de $r$ para especies de alta resiliencia entre 0,6 y 1,5 y error de observación y para evaluar la compatibilidad de los valores de $\mathrm{r}$ y k con las series de capturas y las definiciones de biomasa al inicio y final de las series, los pares de valores r-k se evaluaron con una distribución de Bernoulli. En los casos en que las combinaciones de $\mathrm{r}$ y $\mathrm{k}$ conducen al colapso de la población o excedan la capacidad de carga antes del final de la serie de tiempo, se les asignó cero y fueron desechados. En cambio las combinaciones de $\mathrm{r}$ y $\mathrm{k}$ que dieron valores de biomasa final (como proporción de $\mathrm{k}$ ) entre valores de $\lambda_{1} \mathrm{y} \lambda_{2}$, se les asigna el valor de uno y fueron seleccionados como verosímiles. Después de una primera iteración se redefinen los intervalos de $\mathrm{r}$ y $\mathrm{k}$ y se vuelve a repetir el proceso en una segunda iteración.

Para cada par de valores de $\mathrm{r}$ y $\mathrm{k}$ elegidos como verosímiles en la segunda iteración, se estimaron las siguientes cantidades de manejo:

$$
M R S=\frac{r k}{4}, B_{M R S}=\frac{k}{2}, F_{M R S}=\frac{r}{2}
$$

Se calculó la media geométrica de $\mathrm{r}, \mathrm{k}, \mathrm{MRS}, \mathrm{B}_{\mathrm{MRS}}$ (biomasa que genera el MRS), $\mathrm{F}_{\text {MRS }}$ (mortalidad de pesca en el MRS) y Bt para cada año de todas las series de tiempo. Como un intervalo de confianza al 95\% de las cantidades de manejo, se estimó con el antilogaritmo de la suma y resta de dos veces la desviación estándar a la media de los logaritmos.
Para realizar el análisis de riesgo de considerar algún escenario de cuota de captura para el manejo de la pesquería, se usaron las estimaciones de biomasa de 2012 y los respectivos valores de $\mathrm{r}$ y $\mathrm{k}$ que resultaron verosímiles, según el método de Martell \& Froese (2012), para el conjunto de las 7 zonas y se proyectó la biomasa hacia el 2018 con el modelo de Schaefer, considerando escenarios de captura anual inferiores al MRS, y después, para cada escenario de captura, se estimó el porcentaje de las ocasiones en que la biomasa se colapsó, se mantuvo mayor a cero pero inferior a la $\mathrm{B}_{\mathrm{MRS}} \mathrm{y}$ las veces en que resultó mayor que la $\mathrm{B}_{\mathrm{MRS}}$.

\section{RESUltados}

Para las 7 zonas analizadas los valores de $r$ variaron entre 0,9 y 1,4, los cuales son relativamente altos, típicos de especies altamente resilientes. Los valores de capacidad de carga $\mathrm{k}$ variaron entre 390 y 14.814 t, el MRS de 131 a 3.302 t, la $\mathrm{B}_{\mathrm{MRS}}$ de 195 a 3.788 t y la $\mathrm{F}_{\mathrm{MRS}}$ entre 0,44 y 0,67 , entre las distintas zonas analizadas. Para todo el estado la capacidad de carga estimada fue de $\mathrm{k}=32.550 \mathrm{t}$, el MRS de $7.895 \mathrm{t}, \mathrm{y}$ la $\mathrm{B}_{\mathrm{MRS}}$ de 16.275 t, (Tabla 1, Figs. 2 y 3 ).

El MRS estimado para la zona 1 fue de $1.893 \mathrm{t}$ con un intervalo de confianza al 95\% (IC95\%) entre 1.624 y 2.205 t. Las capturas de jaiba rebasaron el límite superior del MRS en 1996, 2000 y 2008, lo cual provocó la disminución de la biomasa, pero siempre se mantuvo por encima de la $\mathrm{B}_{\mathrm{MRS}}$ estimada (3.788 t) para esta zona. La población de 
Tabla 1. Resultados del método de captura-máximo rendimiento sostenible (MRS) aplicado al stock de jaiba (Callinectes spp.) en Sinaloa, México / Results of the catch-maximum sustainable yield (MRS) method applied to the crab stock (Callinectes spp.) in Sinaloa, Mexico

\begin{tabular}{|c|c|c|c|c|c|c|c|c|c|c|}
\hline \multirow{2}{*}{ Jaiba stock } & \multirow{2}{*}{$\lambda_{01}-\lambda_{02}$} & \multirow{2}{*}{$\lambda_{1}-\lambda_{2}$} & \multirow{2}{*}{$\sigma(v, t)$} & \multicolumn{2}{|c|}{ Segunda iteración } & \multicolumn{5}{|c|}{ Media geométrica (Intervalos de confianza 95\%) } \\
\hline & & & & $\mathrm{r}_{1}$ & $\mathrm{k}_{1}$ & $\mathrm{r}$ & k & MRS & BMRS & FMRS \\
\hline \multirow[t]{2}{*}{ Zona 1} & $0,5-0,9$ & $0,3-0,7$ & 0 & $0,6-1,8$ & $4938-10624$ & 0,999 & 7576 & 1893 & 3788 & 0,499 \\
\hline & & & & & & $(0,612-1,632)$ & $(4923-11659)$ & $(1624-2205)$ & $(2461-5829)$ & $(0,306-0,816)$ \\
\hline \multirow[t]{2}{*}{ Zona 2} & $0,5-0,9$ & $0,3-0,7$ & 0 & $0,6-1,8$ & $525-920$ & 1,42 & 724 & 206 & 362 & 0,571 \\
\hline & & & & & & $(0,695-1,877)$ & $(532-986)$ & $(151-283)$ & $(266-493)$ & $(0,348-0,939)$ \\
\hline \multirow[t]{2}{*}{ Zona 3} & $0,5-0,9$ & $0,01-0,4$ & 0 & $0,6-1,5$ & $11137-17318$ & 0,892 & 14814 & 3302 & 7407 & 0,446 \\
\hline & & & & & & $(0,573-1,386)$ & (11768-18647) & $(2657-4104)$ & (5884-9323) & $(0,287-0,693)$ \\
\hline \multirow[t]{2}{*}{ Zona 4} & $0,5-0,9$ & $0,3-0,7$ & 0 & $0,6-1,8$ & $4747-8572$ & 1,109 & 6653 & 1844 & 3326 & 0,555 \\
\hline & & & & & & $(0,681-1,806)$ & (4775-9268) & $(1418-2399)$ & $(2387-4634)$ & $(0,341-0,902)$ \\
\hline \multirow[t]{2}{*}{ Zona 5} & $0,5-0,9$ & $0,3-0,7$ & 0 & $0,6-1,8$ & $1294-2146$ & 0,976 & 1741 & 424 & 870 & 0,488 \\
\hline & & & & & & $(0,617-1,544)$ & $(1312-2310)$ & $(344-523)$ & $(656-1155)$ & $(0,309-0,772)$ \\
\hline \multirow[t]{2}{*}{ Zona 6} & $0,5-0,9$ & $0,3-0,7$ & 0 & $0,6-1,8$ & $332-494$ & 1,352 & 390 & 131 & 195 & 0,676 \\
\hline & & & & & & $(0,803-2,276)$ & $(320-474)$ & $(86-201)$ & $(160-237)$ & $(0,401-1,138)$ \\
\hline \multirow[t]{2}{*}{ Zona 7} & $0,5-0,9$ & $0,01-0,4$ & 0 & $0,6-1,8$ & $1594-2474$ & 0,970 & 2048 & 583 & 1024 & 0,570 \\
\hline & & & & & & $(0,634-2,051)$ & $(1562-2684)$ & $(415-820)$ & $(781-1342)$ & $(0,317-1,025)$ \\
\hline \multirow[t]{2}{*}{ Sinaloa } & $0,5-0,9$ & $0,3-0,7$ & 0 & $0,6-1,8$ & $22181-41685$ & 0,970 & 32550 & 7895 & 16275 & 0,485 \\
\hline & & & & & & $(0,612-1,539)$ & $(23118-45831)$ & (6557-9505) & (11559-22915) & $(0,306-0,769)$ \\
\hline
\end{tabular}
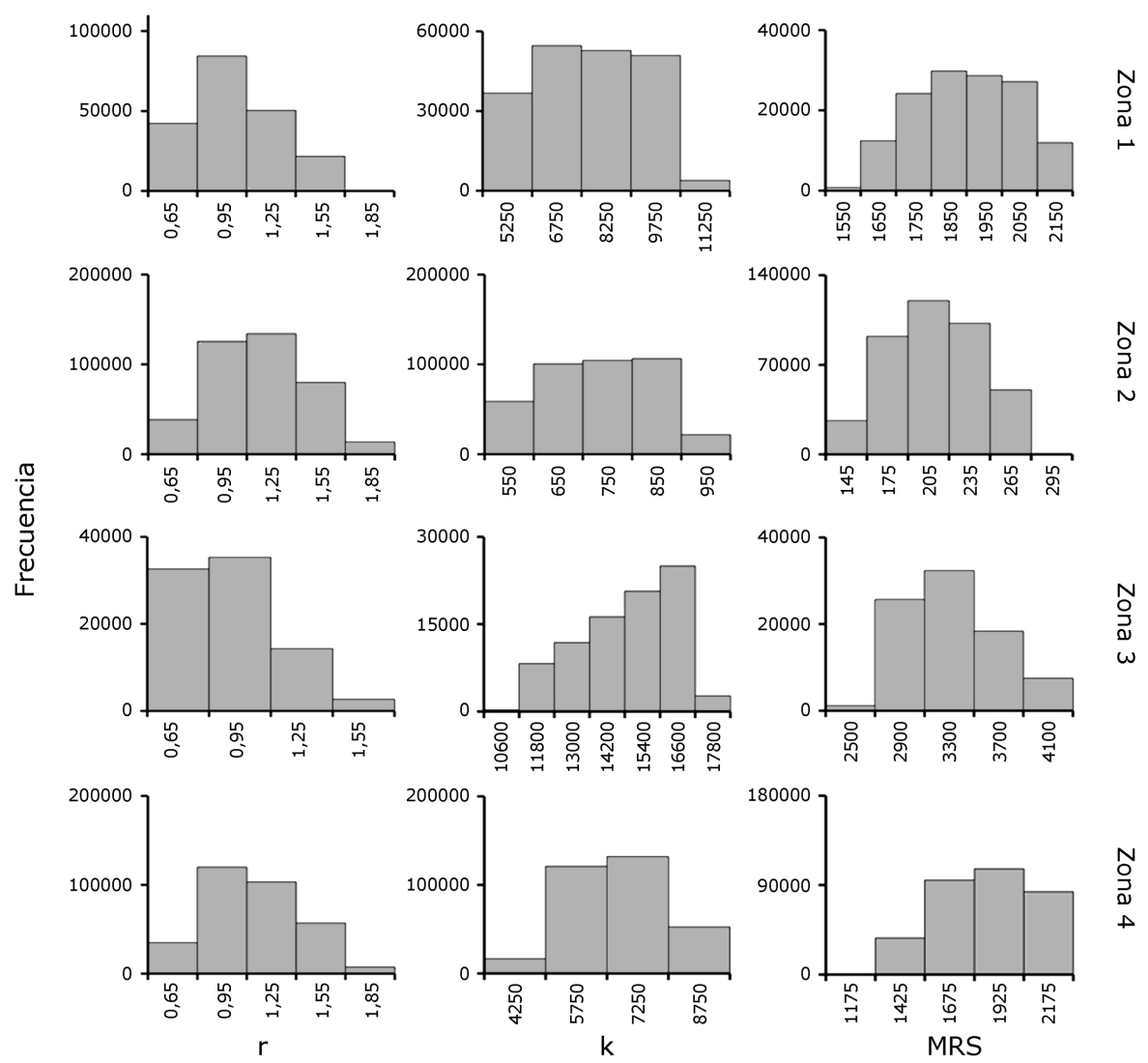

Figura 2. Frecuencias de la capacidad carga (k), tasa máxima del incremento de la población de un stock en un ecosistema ( $r$ ) y máximo rendimiento sostenible (MRS), estimados con el método de captura-MRS para la jaiba en 4 zonas de Sinaloa, México / Frequencies of carrying capacity (k), maximum rate of population increase for a given stock in an ecosystem ( $r$ ) and maximum sustainable yield (MSY) estimates with the catch-MSY method for crab stock in 4 zones of Sinaloa, Mexico 

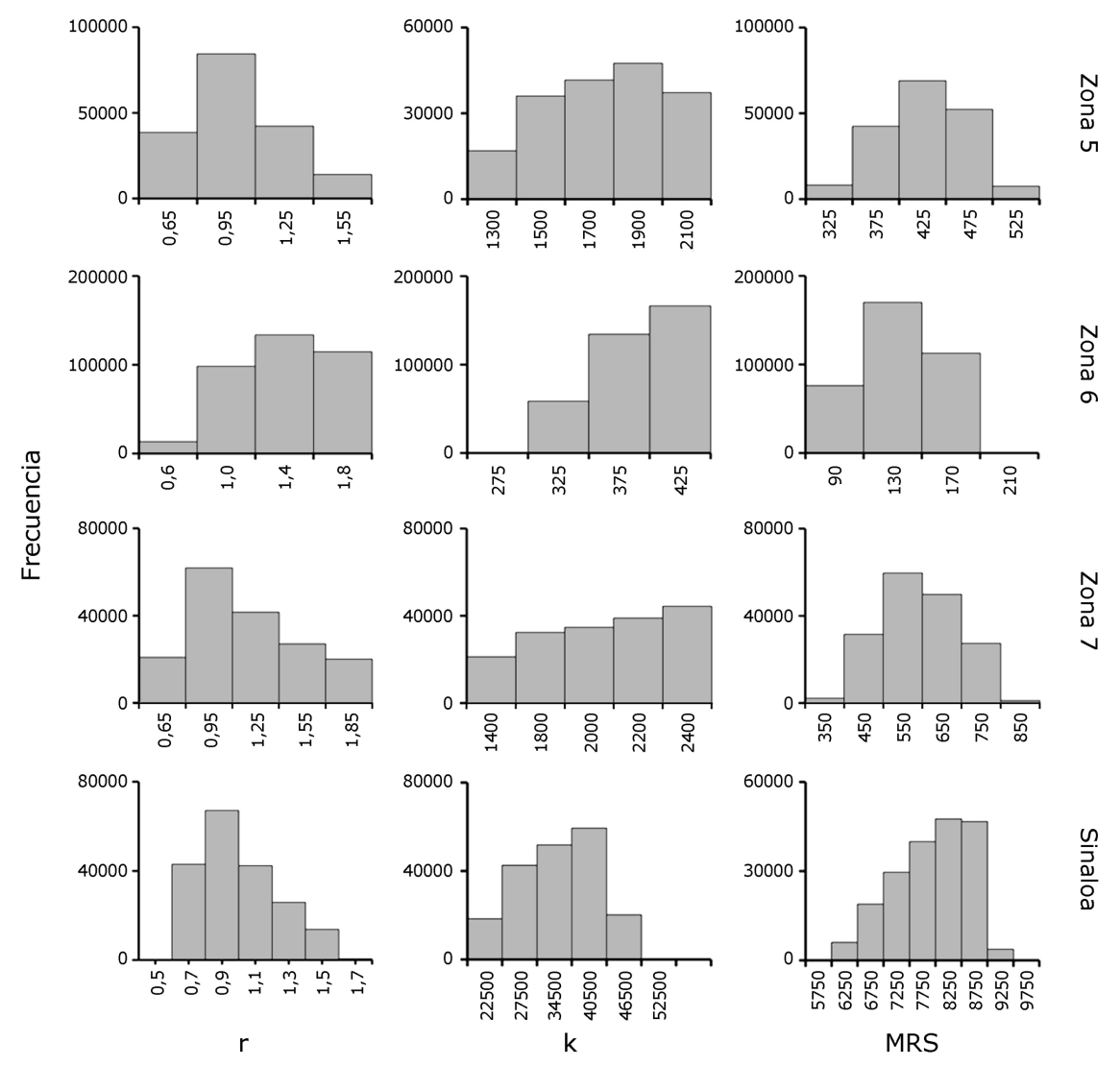

Figura 3. Frecuencias de la capacidad carga (k), tasa máxima del incremento de la población de un stock en un ecosistema ( $r$ ) y máximo rendimiento sostenible (MRS), estimados con el método de captura-MRS para la jaiba en 3 zonas y total de Sinaloa, México / Frequencies of carrying capacity (k), maximum rate of population increase for a given stock in an ecosystem ( $r$ ) and maximum sustainable yield (MSY) estimates with the catch-MSY method for crab stock in 3 zones and total of Sinaloa, Mexico

jaiba puede soportar un incremento en la mortalidad por pesca, pero precautoriamente no se deben incrementar las capturas más allá del MRS. El MRS estimado en la zona 2 fue de $206 \mathrm{t}$ con un IC95\% entre 151 y $283 \mathrm{t}$. Las capturas comerciales de jaiba en esta zona rebasaron el límite superior del MRS en 2009 y 2012, lo cual provocó una caída en la biomasa de la población hasta el límite de la $\mathrm{B}_{\mathrm{MRS}}$, por lo que la población de jaiba en esta zona se encuentra en el máximo nivel de explotación sustentable. En esta zona no se debe permitir que la captura rebase el MRS de $206 \mathrm{t}$ anuales. Para la zona 3 el IC95\% del MRS varió entre 2.657 y 4.104 t y las capturas comerciales rebasaron este indicador en 1995, 1996, 2006, 2007 y 2008, lo cual generó la disminución de la biomasa por debajo de la $\mathrm{B}_{\mathrm{MRS}}(7.407 \mathrm{t})$, por lo que se considera que la población de jaiba en la zona se encuentra a nivel de sobreexplotación. El MRS calculado para la zona 4 fue de 1.844 t con un IC95\% entre 1.418 y 2.399 t. Para los años 2008, 2009 y 2012 las capturas rebasaron el MRS provocando una disminución de la biomasa, pero sin llegar a niveles inferiores de la $\mathrm{B}_{\mathrm{MRS}}(3.326 \mathrm{t})$, por lo que se considera que la población en la zona se encuentra sana. En la zona 5 las capturas comerciales que sobrepasaron el MRS de 424 t (IC95\% 344-523 t) en el 2010 y 2012 disminuyeron la biomasa hasta el nivel de la $\mathrm{B}_{\mathrm{MRS}}$, por lo que se considera que la población de jaiba en esta zona se encuentra en el máximo nivel de explotación. En la zona 6 las capturas comerciales de 2010 y 2012 fueron mayores al MRS de 131 t (IC95\% 86-201 t) y, aunque la biomasa disminuyó, todavía se mantuvo por encima de la $\mathrm{B}_{\mathrm{MRS}}(195 \mathrm{t})$, por lo que se considera una pesquería relativamente sana. En el caso de la zona 7 las capturas registradas en 2007, 2008 y 2010 estuvieron por encima del límite superior del MRS (583 t IC95\% 415-820t), ocasionando la disminución 
de biomasa por debajo de la $\mathrm{B}_{\mathrm{MRS}}(1.024 \mathrm{t})$, por lo que se considera que el stock de jaiba en la zona se encuentra a nivel de sobre-explotación.

Para todo el estado de Sinaloa el IC95\% del MRS fue estimado entre 6.557 y 9.505 t. En el periodo de 2006 a 2008 las capturas comerciales fueron mayores al límite superior del MRS, provocando la disminución de la biomasa por debajo de la $\mathrm{B}_{\mathrm{MRS}}(16.275$ t) de 2009 a 2011, pero al final de 2012 se recuperó hasta el nivel de la $\mathrm{B}_{\mathrm{MRS}}$, por lo cual se considera que la población de jaiba en Sinaloa se encuentra en el máximo nivel de explotación sustentable (Figs. 4 y 5).
El análisis de riesgo del establecimiento de cuotas de captura para el manejo de la pesquería indica que con $5.000 \mathrm{t}$ anuales se tiene una alta probabilidad (100\%) de que en el 2018 la biomasa de la jaiba se ubique por arriba de la $\mathrm{B}_{\text {MRS }}$ (Fig. 6). Un incremento de la cuota por arriba de 5.000 $t$ anuales, el MRS aumenta rápidamente el riesgo de colapso (hasta un 19\%) cerca del MRS o a desarrollar una biomasa inferior a la $\mathrm{B}_{\mathrm{MRS}}(35 \%)$, mientras que reduce la probabilidad $(46 \%)$ de alcanzar una biomasa superior a la $\mathrm{B}_{\mathrm{MRS}}$.

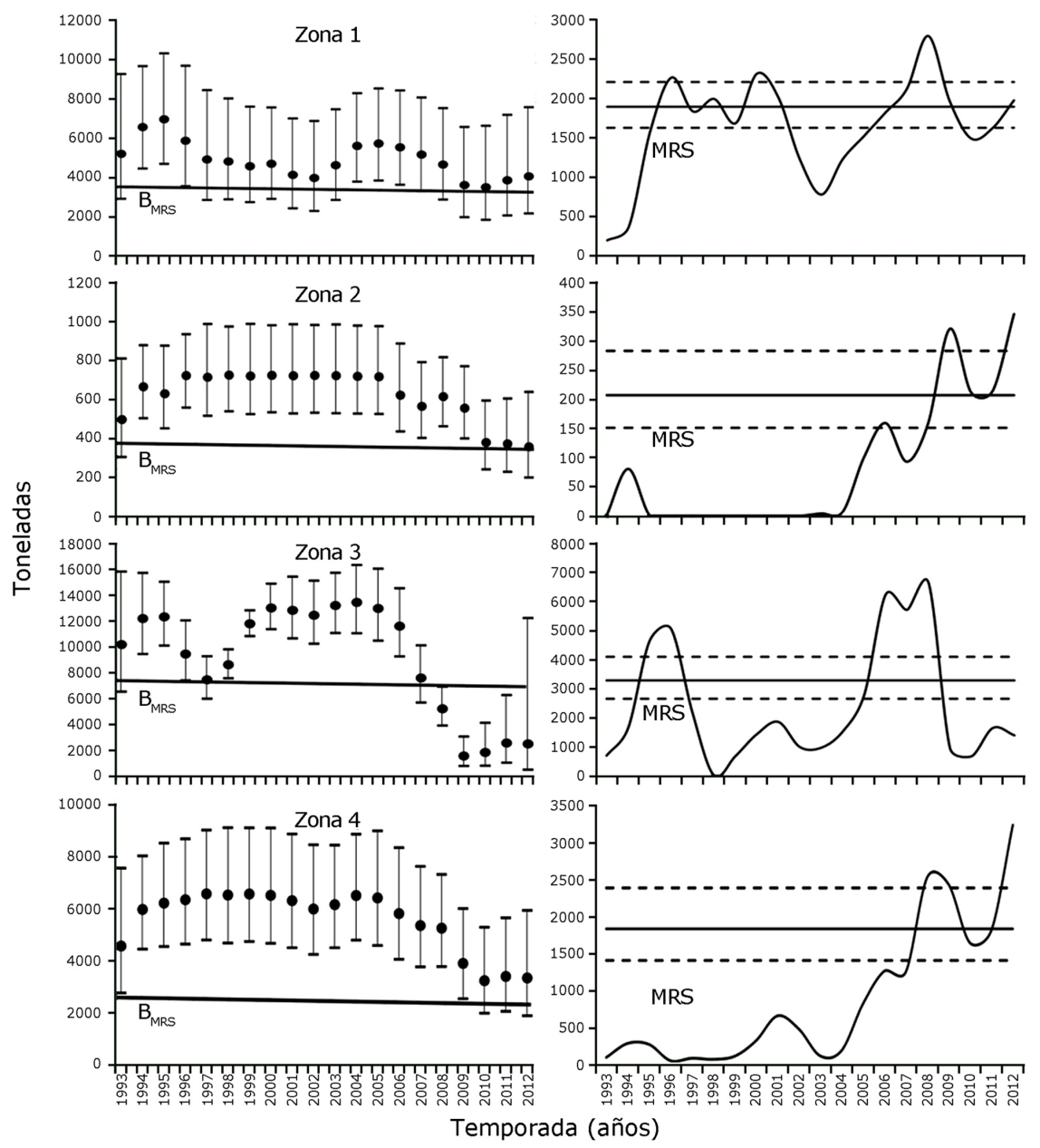

Figura 4. Estimaciones de biomasa de la jaiba con un intervalo de confianza al $95 \%$ (la línea indica la biomasa al máximo rendimiento sostenible, $B_{\text {MRS }}$ ) y captura con la media del MRS con un intervalo de confianza al 95\%, en 4 zonas de Sinaloa, Mexico (1993-2012) / Crab biomass estimates with a $95 \%$ confidence intervals (the line indicates the biomass at the maximum sustainable yield, $\mathrm{B}_{\mathrm{MSY}}$ ) and capture with the MSY mean with a $95 \%$ confidence interval, in 4 areas of Sinaloa, Mexico (1993-2012) 


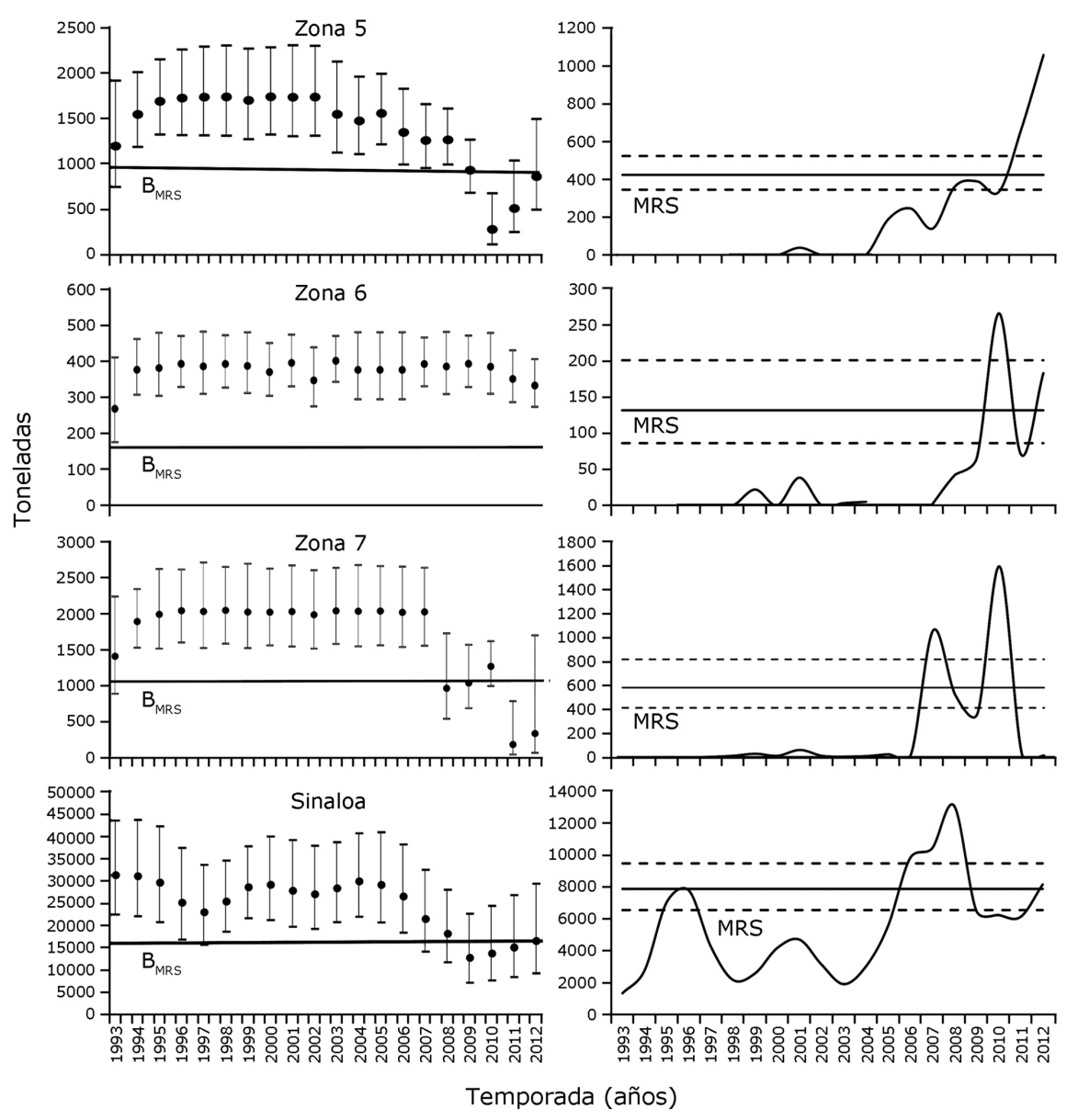

Figura 5. Estimaciones de biomasa de la jaiba con un intervalo de confianza al 95\% (la línea indica la biomasa al máximo rendimiento sostenible, $B_{\text {MRS }}$ ) y captura con la media del MRS con un intervalo de confianza al 95\%, en 3 zonas y total de Sinaloa, Mexico (1993-2012) / Crab biomass estimates with a $95 \%$ confidence intervals (the line indicates the biomass at the maximum sustainable yield, $\mathrm{B}_{\mathrm{MSY}}$ ) and capture with the MSY mean with a 95\% confidence interval, in 3 zones and total of Sinaloa, Mexico (1993-2012)

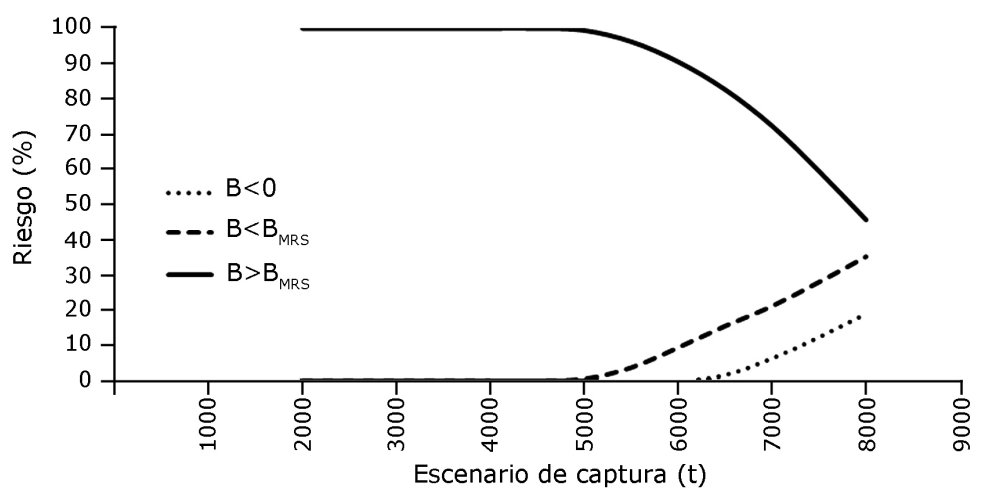

Figura 6. Análisis de riesgo calculado para Sinaloa, México, asumiendo varios escenarios de captura inferiores a 8.000 t de jaiba (máximo rendimiento sostenible, MRS, calculado por el método de captura-MRS) / Risk analysis estimated for Sinaloa, Mexico, assuming several capture scenarios of less $8,000 \mathrm{t}$ of crab (maximum sustainable yield, MSY, calculated by the catch-MSY method) 


\section{Discusión}

Los resultados obtenidos de $\mathrm{r}$, tanto en las 7 zonas como en la global de Sinaloa, se presentaron dentro del rango de alta resiliencia propuesto por Martell \& Froese (2012), indicando una alta capacidad de recuperación de la especie ante una eventual disminución del stock por presión pesquera. Este mismo comportamiento fue reportado por Sutton \& Wagner (2007), Rivera-Velázquez (2013) y Rodríguez-Domínguez et al. (2014) para C. sapidus, $C$. arcuatus y $C$. bellicosus en las costas de Texas, Estados Unidos, Sinaloa y Sonora (México), respectivamente, reportando valores de $r$ entre 0,8 y 1,08, similares a los encontrados en este estudio, pero mayores a lo obtenido por Murphy et al. $(2007)(0,6)$ para C. sapidus en las costas de Florida, Estados Unidos.

La capacidad de carga reportada para la zona 3 se estimó por debajo de lo calculado por Rivera-Velázquez (2013) para la región de Guasave, Sinaloa, México, mientras que para la zona 4 y para la estimación global fue ligeramente mayor que lo obtenido por Rodríguez-Domínguez et al. (2014), quienes realizaron el análisis con series de captura desde 1980 a 2011.

El nivel de rendimiento obtenido de $7.895 \mathrm{t}$, en una $\mathrm{B}_{\mathrm{MRS}}$ de $16.275 \mathrm{t}$, representa un punto de referencia biológico para la especie; sin embargo, en el análisis de riesgo se consideraron valores menores de captura con referencia al MRS calculado por el modelo de Captura-MRS. En este sentido, Martell \& Froese (2012) recomiendan que una cuota precautoria debe ser la del límite inferior (IC del 95\%) del intervalo de confianza del MRS. Sin embargo, dos de las zonas y la estimación global de biomasa estuvieron por debajo de la $\mathrm{B}_{\mathrm{MRS}}$, por lo que se recomienda disminuir las capturas actuales hasta la recuperación del stock poblacional. Esta misma conclusión ha sido reportada anteriormente para el stock de jaiba en Sinaloa por RiveraVelázquez (2013) y Rodríguez-Domínguez et al. (2014), que en años recientes han realizado diversos estudios en diferentes cuerpos lagunares de Sinaloa. Esto difiere a lo mencionado en estudios anteriores para C. arcuatus y C. bellicosus para las mismas zonas, indicando que esta pesquería se encuentra relativamente sana, con valores por debajo de la referencia biológica $(0,5)$, que se considera como la tasa de explotación en la cual se obtiene el MRS (Ortega-Lizárraga 2012, Rodríguez-Domínguez 2012).
La estimación de parámetros como crecimiento, $\mathrm{TM}_{50 \%}$, tasa de explotación y otros son indispensables para el manejo de la pesquería de jaiba (Fischer \& Wolff 2006, Hernández \& Arreola-Lizárraga 2007, Ramos-Cruz 2008, Ortega-Lizárraga 2012), con la finalidad de llevar a la pesquería a un nivel sustentable, que permita mantenerse por muchos años más. Sin embargo, la generación de datos para la evaluación y gestión de las pesquerías son costosas y difíciles de obtener, es aquí donde el método propuesto por Martell \& Froese (2012) alcanza gran notoriedad y utilidad para la evaluación de la pesquería de jaiba, debido a la simplicidad de la técnica.

\section{Agradecimientos}

Gilberto Genaro Ortega Lizárraga agradece a CONACyT, por haber otorgado una beca para culminar el grado de Doctorado, y a la Facultad de Ciencias del Mar, UAS.

\section{LITERATURA CITADA}

Fischer S \& M Wolff. 2006. Fisheries assessment of Callinectes arcuatus (Brachyura, Portunidae) in the Gulf of Nicoya, Costa Rica. Fisheries Research 77: 301-311.

Hernández L \& J Arreola-Lizárraga. 2007. Estructura de tallas y crecimiento de los cangrejos Callinectes arcuatus y C. bellicosus (Decapoda: Portunidae) en la laguna costera Las Guásimas, México. Biología Tropical 55(1): 225-233.

Kennelly SJ \& JP Scandol. 2002. Using a fishery-independent survey to assess the status of a spanner crab Ranina ranina fishery: Univariate analyses and biomass modeling. Crustaceana 75(1): 13-39.

Martell S \& R Froese. 2012. A simple method for estimating MSY from catch and resilience. Fish and Fisheries 14(4): 504-514.

Murphy MD, AL McMillen-Jackson \& B Mahmoudi. 2007. A stock assessment for blue crab, Callinectes sapidus, in Florida waters, 90 pp. Report to the Florida Fish and Wildlife Commission Division of Marine Fisheries Management, Florida Fish and Wildlife Conservation, Commission, Florida Marine Research Institute, St. Petersburg.

Ortega-Lizárraga GG. 2012. Evaluación de la pesquería de la jaiba azul Callinectes arcuatus (Ordway, 1863) de la bahía de Santa María la Reforma. Tesis de Maestría, Facultad de Ciencias del Mar, Universidad Autónoma de Sinaloa, Mazatlán, $50 \mathrm{pp}$.

Ramos-Cruz S. 2008. Estructura y parámetros poblacionales de Callinectes arcuatus Ordway, 1863 (Decapoda: Portunidae), en el sistema lagunar La Joya-Buenavista, Chiapas, México. Julio a diciembre de 2001. Pan-American Journal of Aquatic Sciences 3(3): 259-268. 
Rivera-Velázquez PJ. 2013. Claves de éxito en la cooperativa jaibera Callinectus tortugus en el Municipio de Guasave, Sinaloa. Tesis de Maestría, Facultad de Ciencias del Mar, Universidad Autónoma de Sinaloa, Mazatlán, 87 pp.

Rodríguez-Domínguez G. 2012. Evaluación de la pesquería de jaiba en la bahía de Navachiste, Sinaloa, México, 36 pp. Informe Técnico Final, Comisión Nacional de Áreas Naturales Protegidas, Mazatlán.

Rodríguez-Domínguez G, SG Castillo-Vargasmachuca, R Pérez-González \& EA Aragón-Noriega. 2012. Estimation of individual growth parameters of the brown crab Callinectes bellicosus (Brachyura, Portunidae) using a multi-model approach. Crustaceana 85(1): 55-69.

Rodríguez-Domínguez G, SG Castillo-Vargasmachuca, R Pérez-González \& EA Aragón-Noriega. 2014. CatchMaximum sustainable yield method applied to the crab fishery (Callinectes spp.) in the Gulf of California. Journal of Shellfish Research 33(1): 45-51.
SAGARPA-CONAPESCA. 2011. Anuario estadístico de acuacultura y pesca, 311 pp. Secretaría de Agricultura, Ganadería, Desarrollo Rural, Pesca y Alimentación, Comisión Nacional de Acuacultura y Pesca, Mazatlán.

SAGARPA-CONAPESCA. 2017. Anuario estadístico de acuacultura y pesca, $300 \mathrm{pp}$. Secretaría de Agricultura, Ganadería, Desarrollo Rural, Pesca y Alimentación, Comisión Nacional de Acuacultura y Pesca, Mazatlán.

Sutton G \& T Wagner. 2007. Stock assessment of blue crab (Callinectes sapidus) in Texas coastal waters. Management Data Series 249: 1-53. Texas Parks and Wildlife, Austin.

Recibido el 18 de febrero 2019 y aceptado el 25 de marzo de 2020

Editor: Claudia Bustos D. 